\title{
Calidad de vida relacionada a la salud bucal en escolares de ámbito urbano-marginal
}

\author{
Oral Health Related Quality of Life in schoolchildren of urban-marginal area. \\ Jesús Marcelo-Ingunza ${ }^{1 a}$, César del Castillo-López ${ }^{1 b c}$, Alexis Evangelista-Alva ${ }^{1 b c}$, Pablo Sánchez-Borjas ${ }^{1 c}$
}

\section{RESUMEN}

Objetivos:Medir el impacto de las condiciones orales sobre la Calidad de Vida Relacionada a la Salud Bucal (CVRSB) en escolares de 11 y 12 años de edad, de ámbito urbano-marginal, Pachacútec-Ventanilla, Callao, Lima, durante el año 2013. Materiales y Métodos: Estudio transversal, en 132 escolares de la escuela "Mártir José Olaya”. Se utilizó la versión en español (Perú) del Índice Child-OIDP para evaluar impactos de 17 Condiciones orales sobre 8 Desempeños diarios. Resultados: El 100\% de los escolares entrevistados informaron al menos un desempeño diario afectado. El promedio de condiciones bucales auto-percibidas como problema fue de 4,52 $\pm 1,96$ condiciones. La principal causa de impacto fue Dolor de muela (72,7\%).Comer tuvo la mayor prevalencia de impactos(81,1\%). La mayoría de impactos fue de "muy poca” o "poca”Intensidad. El mayor promedio de Severidad del impacto fue en el desempeño Comer: 1,34 \pm 0,94. La Extensión del impacto alcanzó el promedio de 3,92 $\pm 1,75$ Desempeños impactados. El Índice Child-OIDP global fue 9,71 \pm 7,63. Conclusiones: El estudio mostró que los impactos de las Condiciones orales afectan la calidad de vida de los todos escolares estudiados de Pachacútec-Ventanilla, mayormente en el desempeño Comer, principalmente por causa de Dolor dentario.

PALABRAS CLAVE: Calidad de Vida, salud bucal, CVRSB, urbano-marginal, Child-OIDP.

\section{SUMMARY}

Objectives: To measure the impact of oral conditions on Quality of Life Related to Health (OHRQoL) in schoolchildren aged 11-12 years, at the urban-marginal area, Pachacutec-Ventanilla, Callao, Lima, in 2013. Materials and Methods: A cross sectional study of 132 students of the school "Mártir José Olaya". The Spanish (Peru) Child-OIDP was used to assess the impacts of Oral conditions on daily Performances. Results: $100 \%$ of the stu-

\footnotetext{
${ }^{1}$ Facultad de Estomatología Roberto Beltrán, Universidad Peruana Cayetano Heredia. Lima, Perú.

a Bachiller en Estomatología

b Especialista en Salud Pública.

c Magister en Estomatología.

d Magister en Salud Pública
} 
dents interviewed reported at least one affected daily performance. The average self-perceived oral conditions as the cause of the problem was $4.52 \pm 1.96$ conditions. The main cause of impact was "Toothache" (72.7\%). "Eating" had the highest prevalence of impact (81.1\%). Most impacts were "low intensity". The highest average severity of the impact was on performance Eating $(1.34 \pm 0.94)$. The Extent of the impact reached an average of $3.92 \pm 1.75$ impacted performances. The Child-OIDP overall index was $9.71 \pm 7.63$. Conclusions: The study showed that oral conditions are affecting the quality of life for all schoolchildren studied in Pachacutec-Ventanilla, mostly in "Eating", mainly because of "Toothache".

\section{KEYWORDS: Quality of life, Child-OIDP, marginal-urban,OHRQoL.}

\section{INTRODUCCIÓN}

A la sensación de bienestar que puede ser experimentada por las personas y que representa la suma de sensaciones subjetivas y personales del "sentirse bien” se le ha denominado: "Calidad de vida” (1). La aparición del concepto como tal y la preocupación por la evaluación sistemática y científica del mismo es relativamente reciente, utilizándose en ámbitos muy diversos, como son la salud, la educación, la economía, la política y el mundo de los servicios en general (2).

La calidad de vida relacionada a la salud (CVRS) ha concitado el interés de las instituciones y profesionales de la salud, surgiendo frente a la necesidad de nuevos y mejores indicadores clínicos (3). La Organización Mundial de la Salud hace dos décadas organizó el "WHOQOL group” para el desarrollo de un instrumento para evaluar la calidad de vida aplicable transculturalmente en distintas regiones, edades, sexo, condición socioeconómica (4), para ser usado en la investigación clínica y epidemiológica, tratamientos y evaluación de la atención de salud.El concepto de "Calidad de vida" ha aportado la "percepción del paciente" (subjetiva) en la forma de analizar la situación de salud, permitiendo integrar aspectos de máxima importancia en la vida de las personas, como son la autopercepción de la calidad de vida y la satisfacción del paciente, con las medidas clínicas clásicas (objetivas) de morbilidad, mortalidad y expectativa de vida $(3,5)$.

Los problemas de salud oral más prevalentes, caries dental y enfermedad periodontal, tienen consecuencias no solo físicas, son también económicas, sociales y psicológicas (6), es decir afectan la calidad de vida de los pacientes en gran medida. Debido a las consecuencias que ocasionan las enfermedades orales, repercutiendo en la calidad de vida, entendidas por los impactos intermedios o negativos como: dolor, incomodidad, limitación funcional e insatisfacción con la apariencia,y,por los impactos finales: que afectan el desarrollo de las actividades diarias de las personas, para las que se requiere adecuado rendimiento físico, psicológico y social(7), se hace necesario la medición de cuánto está siendo afectada la calidad de vida, calidad de vida relacionada a la salud oral (CVRSO), mediante indicadores que tengan en cuenta la opinión (percepción) de las personas, es decir, la forma en que percibe su estado de salud-enfermedad (8).

Uno de los indicadores desarrollados para medir la CVRSO es el Índice de Impacto Bucal en las Actividades Diarias de Adultos (Oral Impacts on Daily Performances - OIDP), diseñado para medir la Frecuencia y Severidad de los impactos de condiciones bucales sobre ocho actividades o Desempeños diarios de adultos (9), el que ha sido adaptado para medir la CVRSO en adolescentes (Child-OIDP) (10) y viene usándose en distintitas poblaciones y culturas.

El objetivo del presente trabajo fue medir el impacto de las Condiciones orales (Child-OIDP Index) sobre la Calidad de Vida Relacionada a la Salud Bucal (CVRSB) en escolares del ámbito urbano marginal de Ventanilla, Callao, Lima, en el año 2013.

\section{MATERIAL Y MÉTODOS}

Estudio transversal y observacional. La población estuvo conformada por todos los escolares de $11 \mathrm{y}$ 12 años de edad (nacidos en 2002 y 2001 respectivamente) que estuvieron matriculados en el año 2013, en el nivel primario ó secundario, en la Institución EducativaN 5227 “Mártir José Olaya” de la ciudadela Pachacútec (constituida por una asociación ur- 
bano-marginal de Asentamientos Humanos), distrito de Ventanilla, Callao, Lima, Perú. El tamaño muestral requerido, calculado mediante la fórmula para la estimación de muestreo aleatorio para un 95\% de confianza, fue de 102 escolares, pero se incluyeron en el estudio a 132 escolares que cumplieron con los criterios de selección (entre 11 y 12 años de edad, de ambos sexos, con Consentimiento informado de los padres y Asentimiento informado del escolar, aparentemente sanos y sin alguna alteración crónica sistémica).

Para iniciar el trabajo de campo se tuvo reuniones informativas con los padres de familia, para informar sobre el estudio y solicitar la autorización para incluir a sus hijos(as) en el estudio. Con aquellos escolares cuyos padres autorizaron la participación de su hijo(a) en el estudio, mediante un Consentimiento informado, la muestra quedó conformada, según sexo por 74 mujeres y 58 varones y según nivel educativo por 104 escolares de primaria y 28 de secundaria. La recolección de datos se realizó en los meses de Agosto y Setiembre de 2013, utilizando la versión validada en español (Perú) del Índice Child-OIDP $(12,13)$. Primero, mediante el cuestionario auto-administrado a los escolares, en grupos de 08 a 10 escolares, en aulas y horarios de la institución educativa, para que cada escolar identifique, de la lista de 17 Condiciones bucales, aquellas que habían ocasionado problema o impacto para sus actividades cotidianas o Desempeños, en los últimos tres meses. Luego, con cada escolar se tuvo la entrevista estructurada individual, cara a cara, con duración de ocho a doce minutos, efectuada por el investigador, previamente capacitado por otro investigador con experiencia (13), para recoger los datos de Frecuencia, Severidad, Extensión y Severidad de los impactos de las condiciones orales.

Para el dato de Frecuencia de los impactos en los Desempeños diarios, se preguntó a cada escolar, cuál (les) de las 17 condiciones bucales, señaladas previamente en el cuestionario auto-administrado, habían causado molestias en cada uno de los ocho Desempeños habituales, y cuán a menudo en los últimos 3 meses habían sucedido. Para la Severidad de los impactos en los Desempeños, se preguntó a cada escolar cuán severo había sido el impacto en cada desempeño. La Frecuencia y la Severidad fueron registradas, según el esquema usado por Gherunpong $(10,14)$ en una escala de 1 a 3 en cada caso.
Los puntajes del Índice Child-OIDP, o impacto de las Condiciones bucales en cada Desempeño diario del escolar, se calcularon multiplicando los puntajes de Frecuencia por Severidad del impacto para cada Desempeño habitual, en un rango de 0-9, multiplicando luego el resultado por 100 y dividiéndolo entre 9. El puntaje global del Índice Child-OIDP por escolar se calculó sumando los puntajes del índice en las ocho actividades, en un rango de 0-72, multiplicándolo luego por 100 y dividiéndolo entre 72. La Extensión del impacto de las Condiciones bucales en los Desempeños se obtuvo determinando el número máximo de Desempeños impactados por escolar, en un rango de 0-8, y para la muestra se promedió el número de Desempeños impactados entre los escolares $(11,13,14)$. Para evaluar la Intensidad de los impactos en cada Desempeño, los puntajes del Índice fueron clasificados en una escala de cinco categorías, desde "Intensidad muy severa" hasta "Muy poca Intensidad”, según propuesto por Gherunpong (14). Se analizó la frecuencia de Condiciones orales percibidas como causa de impacto y la frecuencia de Desempeños impactados, según sexo y según nivel educativo, mediante la prueba Chi-cuadrado. La comparación de medias de Condiciones orales percibidas como causa de impacto, de medias de la Extensión del impacto en los Desempeños y de medias de la Severidad del impacto en los Desempeños, según sexo y según nivel educativo, se realizó utilizando la Prueba U de Mann-Whitney.

Para este estudio se tuvo previamente la aprobación del Comité Institucional de Ética de la Universidad Peruana Cayetano Heredia (Registro SIDISI: 61171, Aprobación CIE-UPCH: 27-Feb-2013) y de la institución educativa. Los escolares incluidos en el estudio tuvieron el Consentimiento informado firmado por el padre o madre y el Asentimiento firmado por el escolar.

\section{RESULTADOS}

La prevalencia de impactos de las Condiciones orales en los Desempeños diarios en escolares de Pachacútec-Ventanilla, fue muy Alta: 100,0 \% de escolares con al menos un impacto en alguno de los Desempeños diarios (Child-OIDP $>0$ ) (Tabla 1). Los Desempeños con mayor prevalencia de impactos fueron: Comer (81,1 \%), Limpiarse los dientes (66,7 \%), Dormir (46,2 \%) y Hablar (43,2 \%). Los Desempeños 
con menor prevalencia de impactos fueron Socializar (34,8 \%) y Sonreír (37,1 \%).

Las condiciones bucales mencionadas como causa de impacto fueron 16 de una lista de 17 condiciones. Las mencionadas con mayor frecuencia fueron dolor de muelas (72,7\% de los 132 escolares) y Erupción de diente permanente $(54,6 \%)$. Fractura de diente permanente $(7,0 \%)$ fue la menos mencionada y la condición deformidad de la boca o cara no fue mencionada por ningún escolar (Tabla 2). En promedio cada escolar mencionó 4,52 \pm 1,96 condiciones como causas de impacto y en los escolares de nivel educativo primario el promedio fue significativamente mayor que en los del nivel secundario $(p=0,047)$. La condición Erupción de diente permanente tuvo un promedio significativamente mayor en escolares de primaria que en los de secundaria ( $\mathrm{p}<0,001)$ y así mismo, mayor en mujeres que en varones $(p=0,004)$ (Tabla 2$)$.

La Intensidad del impacto de las condiciones orales sobre todos los Desempeños diarios en los 132 escolares entrevistados, alcanzó las frecuencias más altas en las categorías de "Muy poca Intensidad" (61,4\% de los entrevistados) y "Poca Intensidad" (19,7\%), y en la categoría "Severa Intensidad” alcanzó solo el 2,3\% (Tabla 1). Las categorías de Intensidad "Muy poca” y "Poca", sumadas, tuvieron las frecuencias más altas en los Desempeños Socializar (86,9 \% de los escolares) y Hablar (77,2 \%), y la frecuencia más baja en el desempeño Comer (61,6 \%). Las categorías de Intensidad "Severa” y "Muy Severa”, sumadas, tuvieron las frecuencias más altas en los Desempeños Dormir (16,4 \%) y Estudiar (14,6 \%), y la frecuencia más baja en el desempeño Socializar (4,4 \%), (Tabla No 1 ).

La Extensión de impactos tuvo un promedio de $3,92 \pm 1,75$ Desempeños impactados en cada escolar de la muestra de 132 escolares (mínimo 1 y máximo 8 Desempeños impactados). El promedio fue mayor en varones que en mujeres y mayor en escolares de nivel Primaria que Secundaria, sin diferencia significativa (Tabla 3).

Los Desempeños con mayor Frecuencia de impactos fueron Comer (81,1\% de los escolares de la muestra), Limpiarse la boca (66,7\%) y Dormir (46,2 $\%)$. Socializar tuvo la menor proporción de impactos (34,8\%). Los Desempeños Dormir y Socializar tuvieron significativamente mayor proporción de impactos en los escolares del nivel educativo Primaria que en los de Secundaria ( $\mathrm{p}=0,002$ y $\mathrm{p}=0,001$, respectivamente) (Tabla 4). No se encontró diferencias significativas en la frecuencia de los impactos en los Desempeños según sexo.

El puntaje promedio de Severidad del impacto de las condiciones orales sobre los Desempeños, tuvo los valores más altos en los Desempeños Comer (1,34 $\pm 0,94)$ y Limpiarse la boca $(1,03 \pm 0,96)$, y el menor valor en Socializar $(0,51 \pm 0,80)$ (Tabla 1$)$. En el desempeño Comer el promedio de Severidad fue significativamente mayor en escolares del nivel educativo secundaria que en los del nivel primaria $(\mathrm{p}=0,014)$; en los Desempeños Dormir, Emoción, Estudiar y Socializar la Severidad fue significativamente mayor en los escolares de Primaria que en los de Secundaria. Ningún desempeño tuvo promedios de Severidad de impacto significativamente diferentes según sexo (Tabla 5).

El puntaje promedio del índice Child-OIDP en la muestra (132 escolares) fue 9,71 \pm 7,63(rango entre 1 a 38, de un máximo posible de 72). El promedio del Child-OIDP más alto lo tuvo el desempeño Comer $(2,11 \pm 1,92)$ seguido por el desempeño Limpiarse los dientes $(1,69 \pm 2,12$ ) (rango entre 0 a 9 , de un máximo posible de 9). Los menores puntajes Child-OIDP los tuvieron el desempeño Socializar $(0,63 \pm 1,21)$ (rango entre 0 a 9, de un máximo posible de 9) y Hablar $(0,94 \pm 1,39$ ) (rango entre 0 a 6 , de un máximo posible de 9$)$.

\section{DISCUSIÓN}

En este estudio realizado en el ámbito urbano-marginal de Ventanilla, Callao, Lima, la totalidad de la muestra estudiada, 132 escolares, manifestó al menos un impacto que afectó sus actividades diarias durante los últimos 3 meses (Child-OIDP $>0$ ). Entre los estudios realizados en el Perú con el mismo Índice, la alta prevalencia de impactos de las condiciones bucales hallada en este estudio: 100,0 \%, es igual a la hallada en el distrito de San Juan de Miraflores-Lima: 100,0 $\%$ (15) y superior a la encontrada en Zapallal, Puente Piedra, Lima: 82,0 \%(13); es similar a la hallada en ámbito urbano de Abancay (2300 msnm.), Apurímac:99,2 \% (16), y es superior a la hallada en Canchaque y San Miguel del Faique, (1196 msnm.) en ámbito rural de Huancabamba, Piura: 88,7 \% (11). Así 
Tabla $N^{\circ}$ 1. Prevalencia, intensidad y puntaje Child-OIDP de los impactos orales en los escolares de la Ciudadela Pachacutec, 2013

\begin{tabular}{|c|c|c|c|c|c|c|c|c|c|}
\hline $\begin{array}{c}\text { Impactos Orales } \\
\text { en las Actividades } \\
\text { Diarias }\end{array}$ & $\begin{array}{l}\text { Todos las } \\
\text { actividades } \\
\text { diarias }\end{array}$ & Comer & Hablar & $\begin{array}{l}\text { Limpiarse } \\
\text { los dientes }\end{array}$ & Dormir & Emoción & Sonreir & Estudiar & Socializar \\
\hline Prevalencia (\%) & 100,0 & 81,1 & 43,2 & 66,7 & 46,2 & 41,7 & 37,1 & 41,7 & 34,8 \\
\hline$n=132$ & 132 & 107 & 57 & 88 & 61 & 55 & 49 & 55 & 46 \\
\hline \multicolumn{10}{|c|}{ Puntaje de Severidad de impactos $n=132$} \\
\hline Rango: mín.-máx. & $1-20$ & $0-3$ & $0-3$ & $0-3$ & $0-3$ & $0-3$ & $0-3$ & $0-3$ & $0-3$ \\
\hline Media (d.e.) & $6,20(3,73)$ & $1,34(0,94)$ & $0,61(0,78)$ & $1,03(0,96)$ & $0,80(1,03)$ & $0,64(0,88)$ & $0,58(0,88)$ & $0,70(0,98)$ & $0,51(0,80)$ \\
\hline
\end{tabular}

Intensidad de Impactos (\% de escolares con impactos $n=132$

\begin{tabular}{|c|c|c|c|c|c|c|c|c|c|}
\hline Muy роса & 61,4 & 30,8 & 33,3 & 39,8 & 31,1 & 43,6 & 42,9 & 41,8 & 54,3 \\
\hline Poca & 19,7 & 30,8 & 43,9 & 27,3 & 26,2 & 25,5 & 28,6 & 21,8 & 32,6 \\
\hline Moderada & 16,7 & 27,1 & 15,8 & 21,6 & 26,2 & 23,6 & 16,3 & 21,8 & 8,7 \\
\hline Severa & 2,3 & 8,4 & 7,0 & 4,5 & 8,2 & 5,5 & 4,1 & 7,3 & 2,2 \\
\hline Muy Severa & 0,0 & 2,8 & 0,0 & 6,8 & 8,2 & 1,8 & 8,2 & 7,3 & 2,2 \\
\hline go: mín.-máx. & $1-38$ & $0-9$ & $0-6$ & $0-9$ & $0-9$ & $0-9$ & $0-9$ & $0-9$ & $0-9$ \\
\hline Media (d.e.) & $9,71(7,63)$ & $2,11(1,92)$ & $0,94(1,39)$ & $1,69(2,12)$ & $1,35(2,14)$ & $0,95(1,55)$ & $0,95(1,86)$ & $1,11(1,96)$ & $0,63(1,21)$ \\
\hline
\end{tabular}

d.e. Desviación Estándar

mín.-máx.: mínimo-máximo 
Tabla $N^{\circ}$ 2. Frecuencia y media de condiciones orales percibidas como causa de impactos orales según sexo y nivel educativo en escolares de la Ciudadela Pachacutec. 2013 ( $N=132)$

\begin{tabular}{|c|c|c|c|c|c|c|c|c|}
\hline \multirow{2}{*}{$\begin{array}{l}\text { Condiciones orales percibidas } \\
\text { como causa de Impactos }\end{array}$} & & & \multicolumn{3}{|c|}{ Sexo } & \multicolumn{3}{|c|}{ Nivel Educactivo } \\
\hline & \multicolumn{2}{|c|}{ Frecuencia } & Mujeres & \multicolumn{2}{|c|}{ Varones $(n=58)$} & \multirow{2}{*}{$\begin{array}{c}\begin{array}{c}\text { Primaria } \\
(\mathbf{n}=104)\end{array} \\
\% \%\end{array}$} & \multirow{2}{*}{$\begin{array}{c}\begin{array}{c}\text { Secunda- } \\
\text { ria }(\mathbf{n}=28)\end{array} \\
\%\end{array}$} & \multirow[b]{2}{*}{$\mathrm{p}^{*}$} \\
\hline & $\mathrm{n}$ & $\%$ & $\%$ & $\%$ & $\mathrm{p}^{*}$ & & & \\
\hline Dolor de muela & 96 & 72,7 & 71,6 & 74,1 & 0,749 & 75,0 & 64,3 & 0,130 \\
\hline Diente sensible & 50 & 37,9 & 36,5 & 39,7 & 0,647 & 37,5 & 39,3 & 0,569 \\
\hline Diente cariado & 49 & 37,1 & 32,4 & 43,1 & 0,897 & 34,6 & 46,4 & 0,874 \\
\hline Caída de un diente de leche & 55 & 41,7 & 41,9 & 41,4 & 0,477 & 40,4 & 46,4 & 0,716 \\
\hline Dientes separados & 34 & 25,8 & 20,3 & 32,8 & 0,948 & 26.9 & 21,4 & 0,277 \\
\hline Fractura de diente permanente & 9 & 7,0 & 2,7 & 12,1 & 0,044 & 6,7 & 7,1 & 0,530 \\
\hline Color de dientes & 24 & 18,3 & 18,9 & 17,2 & 0,401 & 23,1 & 0,0 & n.a. \\
\hline Forma o tamaño de los dientes & 18 & 13,7 & 12,2 & 15,5 & 0,708 & 14,4 & 10,7 & 0,306 \\
\hline Posición de los dientes & 28 & 21,2 & 18,9 & 24,1 & 0,766 & 18,3 & 32,1 & 0,951 \\
\hline Sangrado de encias & 36 & 27,2 & 31,1 & 22,4 & 0,133 & 26,0 & 32,1 & 0,740 \\
\hline Inflamación de las encías & 32 & 24,2 & 24,3 & 24,1 & 0,489 & 26,0 & 17,9 & 0,188 \\
\hline Sarro o cálculo dental & 20 & 15,1 & 6,8 & 25,9 & 0,999 & 15,4 & 14,3 & 0,443 \\
\hline Llagas o heridas en la boca & 27 & 20,3 & 16,2 & 25,9 & 0,915 & 22,1 & 14,3 & 0,182 \\
\hline Mal aliento & 25 & 19,1 & 12,2 & 27,6 & 0,987 & 21,2 & 10,7 & 0,104 \\
\hline Erupción de diente permanente & 72 & 54,6 & 64,9 & 41,4 & 0,004 & 66,3 & 10,7 & $<0,001$ \\
\hline Pérdida de diente permanente & 21 & 15,3 & 14,9 & 17,2 & 0,640 & 18,3 & 7,1 & 0,075 \\
\hline Todas las condiciones orales & 132 & 100,0 & 100,0 & 100,0 & & 100,0 & 100,0 & \\
\hline $\begin{array}{l}\text { Media de condiciones percibidas } \\
\text { como causa de impacto }\end{array}$ & \multicolumn{2}{|c|}{$4,52(1,96)$} & $4,83(2,12)$ & $4,27(1,80)$ & $0,121 * *$ & $4,71(2,09)$ & $3,79(1,13)$ & $0,047 * *$ \\
\hline Media (d.e.) & & & & & & & & \\
\hline
\end{tabular}

n.a. No aplicable

d.e. Desviación Estándar

* Prueba Comparación de Proporciones

** Prueba U de Mann-Whitney

Tabla 3. Extensión del impacto de las condiciones orales sobre los desempeños diarios en escolares de 11 y 12 años de la Ciudadela Pachacutec según sexo y nivel educativo. 2013.

\begin{tabular}{|c|c|c|c|c|c|}
\hline & \multirow{2}{*}{$\mathbf{n}$} & \multirow{2}{*}{ Media (d.e.) } & \multicolumn{2}{|c|}{ Rango } & \multirow{2}{*}{$\mathbf{p}$} \\
\hline & & & Minimo & Maximo & \\
\hline \multicolumn{6}{|l|}{ Sexo } \\
\hline Mujeres & 74 & $3,70(1,69)$ & 1 & 8 & \multirow{2}{*}{0,11} \\
\hline Varones & 58 & $4,19(1,79)$ & 2 & 8 & \\
\hline \multicolumn{6}{|l|}{ Nivel educativo } \\
\hline Primaria & 104 & $4,08(1,86)$ & 1 & 8 & \multirow{2}{*}{0,07} \\
\hline Secundaria & 28 & $3,32(1,05)$ & 2 & 6 & \\
\hline Total & 132 & $3,92(1,75)$ & 1 & 8 & \\
\hline
\end{tabular}


Tabla 4. Frecuencia de desempeños diarios con impactos de condiciones orales en escolares de 11 y 12 años de la Ciudadela Pachacutec según sexo y nivel educativo. 2013.

\begin{tabular}{|c|c|c|c|c|c|c|c|c|c|c|c|c|}
\hline \multirow{2}{*}{$\begin{array}{l}\text { Desempeños dia- } \\
\text { rios con impactos }\end{array}$} & \multicolumn{2}{|c|}{$(n=132)$} & \multicolumn{2}{|c|}{$\begin{array}{c}\text { Mujeres } \\
(n=74)\end{array}$} & \multicolumn{2}{|c|}{$\begin{array}{l}\text { Varones } \\
(n=58)\end{array}$} & \multirow[t]{2}{*}{$\mathbf{p}$} & \multicolumn{2}{|c|}{$\begin{array}{c}\text { Primaria } \\
(n=104)\end{array}$} & \multicolumn{2}{|c|}{$\begin{array}{l}\text { Secundaria } \\
\qquad(n=28)\end{array}$} & \multirow[t]{2}{*}{$\mathbf{p}$} \\
\hline & $\mathbf{n}$ & $\%$ & $\mathbf{n}$ & $\%$ & $\mathbf{n}$ & $\%$ & & $\mathbf{n}$ & $\%$ & $\mathbf{n}$ & $\%$ & \\
\hline Comer & 107 & 81,1 & 62 & 83,8 & 45 & 77,6 & 0,378 & 80 & 76,9 & 27 & 96,4 & 0,990 \\
\hline Hablar & 57 & 43,2 & 34 & 45,9 & 23 & 39,7 & 0,238 & 47 & 45,2 & 10 & 35,7 & 0,184 \\
\hline Limpiarse la boca & 88 & 66,7 & 46 & 62,2 & 42 & 72,4 & 0,891 & 67 & 64,4 & 21 & 75,0 & 0,854 \\
\hline Dormir & 61 & 46,2 & 34 & 45,9 & 27 & 46,6 & 0,532 & 55 & 52,9 & 6 & 21,4 & 0,002 \\
\hline Emoción & 55 & 41,7 & 26 & 35,1 & 29 & 50,0 & 0,958 & 49 & 47,1 & 6 & 21,4 & 0,007 \\
\hline Sonreir & 49 & 37,1 & 26 & 35,1 & 23 & 39,7 & 0,706 & 39 & 37,5 & 10 & 35,7 & 0,430 \\
\hline Estudiar & 55 & 41,7 & 30 & 40,5 & 25 & 43,1 & 0,618 & 47 & 45,2 & 8 & 28,6 & 0,057 \\
\hline Socializar & 46 & 34,8 & 22 & 29,7 & 24 & 41,4 & 0,919 & 43 & 41,3 & 3 & 10,7 & 0,001 \\
\hline Total & 132 & 100,0 & 74 & 100,0 & 58 & 100,0 & & 104 & 100,0 & 28 & 100,0 & \\
\hline
\end{tabular}

Prueba Chi cuadrado

Tabla 5. Severidad del impacto de las condiciones orales sobre los desempeños en escolares de 11 y 12 años de la Ciudadela Pachacutec según sexo y nivel educactivo. 2013 (n=132)

\begin{tabular}{|c|c|c|c|c|c|c|c|c|c|c|c|c|c|}
\hline \multirow{3}{*}{$\begin{array}{l}\text { Desempeños dia- } \\
\text { rios con impactos }\end{array}$} & \multirow{3}{*}{ Media (d.e.) } & \multicolumn{2}{|c|}{ Rango } & \multirow{2}{*}{\multicolumn{2}{|c|}{$\begin{array}{l}\text { Mujeres } \\
(n=74)\end{array}$}} & \multirow{2}{*}{\multicolumn{2}{|c|}{$\begin{array}{l}\text { Varones } \\
(n=58)\end{array}$}} & \multirow{3}{*}{$\mathbf{p}$} & \multirow{2}{*}{\multicolumn{2}{|c|}{$\begin{array}{l}\text { Primaria } \\
(n=104)\end{array}$}} & \multirow{2}{*}{\multicolumn{2}{|c|}{$\begin{array}{l}\text { Secundaria } \\
\qquad(n=28)\end{array}$}} & \multirow{3}{*}{$\mathbf{p}$} \\
\hline & & Mín. & Máx. & & & & & & & & & & \\
\hline & & & & Media & d.e. & Media & d.e. & & Media & d.e. & Media & d.e. & \\
\hline Comer & $1,34(0,94)$ & 0 & 3 & 1,32 & 0,938 & 1,36 & 0,968 & 0,678 & 1,25 & 0,983 & 1,68 & 0,723 & 0,014 \\
\hline Hablar & $0,61(0,78)$ & 0 & 3 & 0,68 & 0,846 & 0,52 & 0,707 & 0,358 & 0,65 & 0,833 & 0,43 & 0,573 & 0,309 \\
\hline Limpiarse la boca & $1,03(0,96)$ & 0 & 3 & 0,97 & 0,979 & 1,10 & 0,949 & 0,355 & 1,08 & 1,040 & 0,86 & 0,591 & 0,624 \\
\hline Dormir & $0,80(1,03)$ & 0 & 3 & 0,77 & 1,014 & 0,84 & 1,073 & 0,770 & 0,94 & 1,087 & 0,29 & 0,600 & 0,002 \\
\hline Emoción & $0,64(0,88)$ & 0 & 3 & 0,61 & 0,948 & 0,67 & 0,803 & 0,268 & 0,73 & 0,927 & 0,29 & 0,600 & 0,014 \\
\hline Sonreir & $0,58(0,88)$ & 0 & 3 & 0,54 & 0,847 & 0,64 & 0,931 & 0,517 & 0,60 & 0,909 & 0,54 & 0,793 & 0,840 \\
\hline Estudiar & $0,70(0,98)$ & 0 & 3 & 0,72 & 1,027 & 0,67 & 0,925 & 0,969 & 0,80 & 1,046 & 0,32 & 0,548 & 0,044 \\
\hline Socializar & $0,51(0,80)$ & 0 & 3 & 0,36 & 0,610 & 0,69 & 0,977 & 0,079 & 0,62 & 0,862 & 0,11 & 0,315 & 0,002 \\
\hline Total & $6,20(3,73)$ & 1 & 20 & 5,97 & 3,484 & 6,50 & 4,045 & 0,524 & 6,66 & 4,01 & 4,50 & 1,58 & 0,16 \\
\hline
\end{tabular}

d.e.= Desviacion Estándar

Prueba U de Mann-Whitney 
mismo, es superior a la prevalencia hallada con este Índice en otros países: en adolescentes tailandeses: 89,8\% (14), brasileños: 80,7\% (17), Italianos:66,8\% (18), rumanos: 64,9\% (19), sudaneses:54,6\% (20), ingleses:40,4\% (21), y españoles: 30,5\% (22).

La prevalencia de impactos hallada en este estudio, junto con los hallazgos de los otros estudios mencionados en el Perú, confirman prevalencias más altas que las mencionadas en la mayoría de estudios en otros países. Aunque los datos resultan todavía escasos, parecieran indicar un mayor impacto de las condiciones orales en la calidad de vida de adolescentes en poblaciones urbano-marginales y rurales que en poblaciones más urbanas. Ello resultaría coherente también con lo hallado con el Índice OIDP para adultos, en un estudio en Tanzania (2006), pues, se encontró prevalencia de impactos de condiciones orales significativamente mayor en los Desempeños diarios de ancianos de ambiente rural (62,1\%) que de ambiente urbano (48,8 \%) (23).

En el presente estudio, excepto por la condición Deformidad de la boca o cara, todas las demás Condiciones bucales, de la lista de 17 , fueron señaladas por los escolares como causantes de los impactos en sus Desempeños diarios. En promedio fueron 4,52 \pm 1,96 condiciones señaladas por escolar, con promedios significativamente mayores en varones que en mujeres y en escolares de nivel educativo primaria que de secundaria. La Condición oral más frecuentemente mencionada como causa de los impactos fue Dolor de muela, en más de 7 de cada 10 escolares de la muestra (3 de cada 4 escolares, entre los varones y 3 de cada 4 escolares entre los del nivel de educación primaria).Dolor de muela, ha sido hallazgo semejante en la mayoría de los otros estudios en Perú $(12,13,15)$, y guardaría relación con la alta prevalencia de caries en el Perú (para la edad de 12 años, prevalencia de caries: 86,64 \% y CPO-D: 3,67) (24), pues el síntoma frecuente asociado a caries dental avanzada es dolor dentario. Estudios en otros países también han encontrado a Dolor de muela entre las primeras causas de impacto en las actividades diarias de los escolares $(14,18,20,21,22)$.

A la condición Dolor de muela le siguió en frecuencia, como causa de impactos, la condición Erupción de diente permanente: $54,6 \%$, con frecuencia significativamente más alta en escolares mujeres que en varones $\mathrm{y}$ en escolares de nivel primaria que de secundaria. Luego siguió Caída de un diente de leche: 41,7 \%. Esto estaría relacionado con que Erupción de diente permanente o la Caída de un diente de leche, son de naturaleza fisiológica y propias de la edad de los escolares estudiados, aunque en otros estudios no se han encontrado prevalencias tan altas. La pérdida de diente permanente (15,3\%), sarro o cálculo dental $(15,1 \%)$, forma o tamaño de los dientes $(13,7 \%)$ y fractura de diente permanente (7\%), son los problemas orales menos mencionados por los escolares entrevistados en este estudio como causa de impactos.

La Intensidad del impacto de las Condiciones orales sobre la calidad de vida relacionada a la salud bucal (CVRSB), tuvo mayor prevalencia para las categorías "Muy poca” Intensidad y "Poca” Intensidad, por separado y en conjunto para ambas categorías, para todos los Desempeños y para cada desempeño.

La Intensidad del impacto en los Desempeños, en los escolares en este estudio, fue muy consistente con la encontrada en la mayoría de los otros estudios en el país y en otros países, en lo referente a valores más altos en las categorías Muy poca y Poca Intensidad que en las categorías Severa y Muy Severa Intensidad, tanto para el impacto en todos los Desempeños como en los Desempeños específicos de las poblaciones de adolescentes estudiadas (11,13-16). En algunos estudios en otros países se ha encontrado que la categoría Moderada Intensidad de impactos en los Desempeños ha mostrado frecuencia más alta que las otras categorías de Intensidad: en adolescentes de 11 a 16 años de edad en Italia (18) y en ancianos, tanto de ambientes urbanos como de ambientes rurales, en Tanzania (23).

El desempeño Estudiar se ha encontrado entre los Desempeños con valores más altos, cuando son sumadas las categorías Severa y Muy Severa Intensidad de impacto, en el presente estudio, semejante a lo encontrado en el estudio en Zapallal, en población cercana geográficamente, en el Norte de Lima (13), mientras que en los escolares de otros estudios en el país, los valores más altos de las categorías Severa y Muy severa Intensidad han correspondido a alguno de otros Desempeños: Sonreír, Comer y Emoción $(11,16)$. Estos hallazgos parecen indicar que las Condiciones orales impactan con poca Intensidad en todos los Desempeños de los escolares, aunque, en proporciones menores, llegan a impactar con severa Intensi- 
dad a algunos escolares en algunos de los Desempeños.

Las semejanzas entre las Intensidades de impacto en los Desempeños de los escolares del presente estudio y de los otros estudios a nivel nacional, parecen explicarse debido a la similitud de culturas entre los ambientes urbano-marginales y rurales, ya que los pobladores de los asentamientos humanos (urbano-marginales) son provenientes en su mayoría de entornos rurales del interior del país. Algunas diferencias en las frecuencias correspondientes a las Intensidades de impacto "severa y muy severa", en los escolares del presente estudio y de otros países, podrían deberse a diferencias socio-culturales específicas: grado de urbanización, hábitos, costumbres y diferentes prioridades en la vida de los escolares.

La mayoría de escolares estudiados informó impactos en al menos uno, y hasta en ocho Desempeños diarios. Entre los Desempeños más impactados estuvieron Comer y Limpiarse la boca, hallazgos similares a los encontrados en los estudios en el país, en Piura (11), en Lima (15), y en Abancay (16), así como también en algunos estudios en otros países, en Sudán (20), en Tailandia (14) y Brasil (17). Entre los Desempeños menos impactados estuvo Socializar, igualmente a los hallazgos en los otros estudios en el país y en la mayoría de estudios en los otros países. Casi universalmente, en los estudios en adolescentes, con el Child-OIDP, el Desempeño con mayor proporción de impactos corresponde a una importante función del área física de la CVRSB: Comer y disfrutar de la comida, y el Desempeño con menor proporción de impactos corresponde a una función del área psicológica de la CVRSB: Socializar y disfrutar de relacionarse con otros. En poblaciones rurales y urbanas de edades mayores estudiadas con el OIDP, en Tanzania, también se ha encontrado a Comer y Limpiarse la boca como los Desempeños con mayor proporción de impactos y en proporciones superiores a Socializar (23). En el presente estudio, la mayoría de los ocho Desempeños considerados en el Índice tuvieron proporciones de impactos similares según sexo y nivel educativo, pero Dormir y Socializar tuvieron proporciones de impactos significativamente más altas en escolares de nivel primario que de secundaria. Sin embargo, es posible que esta diferencia sea producto de la desproporción en el tamaño de los subgrupos por nivel educativo en la muestra (relación número de escolares de secundaria/escolares de pri- maria: $1 / 3,71)$, antes que un efecto relacionado con el nivel educativo.

Los puntajes de Severidad del impacto de las condiciones orales sobre los Desempeños sirven para calcular los puntajes del Índice, multiplicando Severidad por frecuencia de los impactos, pero también permiten apreciar el grado de impacto auto-percibido por los escolares sobre los Desempeños. En este estudio los puntajes promedio de Severidad de impacto en los Desempeños más altos fueron para los Desempeños Comer y Limpiarse la boca y los menores para Sonreír y Socializar. Los hallazgos para la Severidad del impacto en Comer, Limpiarse la boca y en Socializar son semejantes a los encontrados en los estudios de Zapallal (13), Piura (11) y Abancay (16), en el país. En el presente estudio el promedio de Severidad de impacto en el desempeño Comer fue significativamente mayor en escolares del nivel educativo secundaria que de primaria, y los promedios de Severidad de impacto en los Desempeños Dormir, Emoción, Estudiar y Socializar fueron significativamente mayores en escolares de Primaria que de Secundaria. Sin embargo, las diferencias mencionadas no parecen tener una explicación relacionada con el nivel educativo, pues la diferencia por nivel educativo entre los escolares estudiados es de apenas un grado en el sistema educativo.

La Extensión de los impactos auto-percibidos, es decir, el máximo número de Desempeños impactados por las condiciones orales en los Desempeños de los escolares, tuvo un promedio de 3,92 Desempeños impactados por escolar, en coherencia con que el número de Desempeños impactados en los escolares varió de 1 a 8, es decir, ningún escolar tuvo 0 Desempeños impactados y por tanto la prevalencia de impactos fue de $100 \%$ de escolares con impactos. La Extensión de impactos hallada en este estudio en Pachacútec-Ventanilla, en promedio de casi cuatro Desempeños impactados, es la más alta entre los estudios realizados con el Índice Child-OIDP en el país, superior a la hallada en los estudios en Zapallal: 2,72 Desempeños impactados (13), Abancay: 3,79 (16), Piura:3,01 (11) y San Juan de Miraflores: 1,95 (15).

El puntaje promedio global del Índice Child-OIDP para la muestra de 132 escolares estudiada fue 9,71. Esto es, los escolares estudiados en Pachacútec-Ventanilla, tuvieron una auto-percepción más alta de im- 
pacto, en su calidad de vida relacionada a la salud oral, que los escolares de otras poblaciones estudiadas en Perú: en Zapallal: 7,7 (13) y en Piura: 7,05 (11). El promedio global de impactos también resulta más alto que lo hallado en algunos otros estudios: en Francia: 6,32 (25), en Brasil: 7,1 (26), en Sudan: 1,4 (20), en Italia: 1,9 (18), y cercano a estudios que han encontrado puntajes altos: en Tailandia: 8,85 (14) y en Brasil: $(9,2)(17)$. El puntaje promedio del Índice según Desempeños, muestra que los escolares tuvieron impactos en todos los Desempeños diarios, con los valores más altos en Comer y Limpiarse los dientes y los valores más bajos en Socializar, Sonreír y Emoción. Estos resultados son coherentes con los otros estudios con el Child-OIDP en el país y en otros países, en lo referente a mayores puntajes del índice para los Desempeños Comer y Limpiarse la boca. Así mismo, contribuyen a la conclusión que los escolares, adolescentes, auto-perciben mayor impacto o molestia en sus Desempeños de la dimensión física, y posiblemente valoran menos los impactos o molestias en sus Desempeños de la dimensión social y psicológica.

Ha constituido limitante importante en este estudio la falta de homogeneidad del grupo de escolares incorporados como muestra, ya que no presentaron la misma proporción en los respectivos subgrupos según sexo y nivel educativo; por ello los resultados obtenidos en el presente estudio solo tienen validez interna y no pueden ser aplicados directamente a otras realidades fuera del contexto y entorno donde se desarrolló el estudio.

Se puede concluir que el impacto de las condiciones orales sobre la Calidad de Vida Relacionada a la Salud Bucal (CVRSB) en escolares del ámbito urbano marginal de Ventanilla, Callao, Lima, es muy alto con una prevalencia de $100 \%$ de escolares con impactos, una Severidad de los impactos leve, con poca Intensidad de impacto. El promedio del Índice (Child-OIDP Index) alcanzó un valor alto, mayor que en otros estudios con el mismo índice en el Perú, y según Desempeños tuvo los mayores valores para Comer y Limpiarse la boca. La condición oral que mayormente fue señalada como causa de impactos fue Dolor de muela.

La utilización del Child-OIDP Index para determinar el impacto de las Condiciones orales en la calidad de vida, llega más allá que las evaluaciones epidemiológicas convencionales (solo morbilidad), haciendo más factible la incorporación de la percepción de bienestar o alteración oral de los escolares al diagnóstico de salud poblacional. Esto es, la prevalencia de impactos, el puntaje del Î́ndice Child-OIDP, las condiciones orales identificadas como causas de mayor impacto, los Desempeños identificados como más impactados, pueden complementar los datos epidemiológicos locales para un diagnóstico y planeación de la atención más ajustados a las dimensiones de salud bucal.

\section{Correspondencia:}

Jesús Marcelo Ingunza

Correo electrónico: jesus.marcelo@upch.pe

\section{REFERENCIAS BIBLIOGRÁFICAS}

1. Velarde E, Avila C. Evaluación de la calidad de vida. Salud Pública Mex. 2002; 44:349-61.

2. Gómez M, Sabeh E. Calidad de vida. evolución del concepto y su influencia en la investigación y la práctica. Salamanca: Instituto Universitario de Integración en la Comunidad, Facultad de Psicología, Universidad de Salamanca; 2000.

3. Consiglio E, Belloso W. Nuevos indicadores clínicos: La calidad de vida relacionada con la salud. Medicina (Buenos Aires). 2003 (Citado 26-012015);63(2): 172-8. Disponible en: http://www. scielo.org.ar/scielo.php?script=sci_arttext\&pi$\mathrm{d}=$ S0025-76802003000200014\&lng=es\&nrm=iso

4. WHO-QOL Group. The World Health Organization -Quality of Live: Position paper from the WHO. Soc Sci Med. 1995; 41(10):1403-9.

5. Schwartzmann L. Health-related quality of life: Conceptual aspects. Cienc enferm. 2003; 9(2): 9-21. http:// dx.doi.org/10.4067/S0717-95532003000200002

6. Naito M, Yuasa H, Nomura Y, Nakayama T. Oral health status and health-related quality of life: a systematic review. Journal of Oral Science. 2006; 48:1-7.

7. Locker D. Measuring oral health: a conceptual framework. Community Dent Health. 1988; 5(1):3-18.

8. Pane S, Solans M, Gaite L, Serra-Sutton V. Instrumentos de calidad de vida relacionada con la salud en la edad pediátrica. Revisión sistemática de la literatura: actualización. V Cantabria, España: Departamento de psiquiatría, Hospital Universitario Marqués de Valdecilla, Cantabria; 2006.

9. Adulyanon S, Sheiham A.: Oral impacts on daily performances. En: Slade GD (Editor). Measuring oral health and quality of life. Chapel Hill, NC: University of North Carolina; Dental Ecology 1997. p.151-60. 
10. Gherunpong S, Tsakos G, Sheiham A. Developing and evaluating an oral health-related quality of life index for children; the Child-OIDP. Community Dent Health. 2004; 21:161-9.

11. Del Castillo-López C, Evangelista-Alva A, Sánchez -Borjas P. Impacto de las condiciones bucales sobre la calidad de vida en escolares de ámbito rural, Piura, Perú. Rev Estomatol Herediana. 2014; 24(4):220-8.

12. Bernabé E, Sheiham A, Tsakos G. A comprehensive evaluation of the validity of Child-OIDP: further evidence from Peru. Community Dent Oral Epidemiol. 2008; 36(4):317-25.

13. Sánchez P. Impacto de las condiciones orales sobre la calidad de vida en escolares de 11 y 12 años de edad de Zapallal-Puente Piedra. Lima. Tesis de Maestría. Lima, Perú: Facultad de Estomatología, Universidad Peruana Cayetano Heredia; 2007.

14. Gherunpong S, Tsakos G, Sheiham A: The prevalence and severity of oral impacts on daily performances in Thai primary school children. Health Qual Life Outcomes. 2004; 12: 57.

15. Paredes-Martínez ER, Díaz-Pizán ME. Impacto de las condiciones bucales sobre la calidad de vida en escolares del Distrito de San Juan de Miraflores, Lima, Perú. Rev Estomatol Herediana. 2014; 24(3):171-7.

16. Tineo P. Impacto de las condiciones orales sobre la calidad de vida relacionada a la salud (CVRS) de escolares de 11 y 12 años del ámbito urbano del distrito de Abancay Apurímac en el año 2011.Tesis Maestría. Lima, Perú: Facultad de Estomatología, Universidad Peruana Cayetano Heredia; 2012.

17. Castro R, Cortes M, Leao A, et al. Child-OIDP index in Brazil: Cross-cultural adaptation and validation. Health Qual Life Outcomes. 2008; 6: 68. doi:10.1186/1477-7525-6-68

18. Bianco A, Fortunato L, Nobile CGA, Pavia M. Prevalence and determinants of oral impacts on daily performance: results from a survey among school children in Italy. Eur J Public Health. 2009; 20(5): 595-600.

19. Nucã C, Amariei C, Martoncsak E, TomiD D. Study regarding the correlation between the Child-OIDP index and the dental status in 12-year-old children from Harsova, Constanta County. Oral Health Dent Manag. 2005; 4(4):4-13.

20. Nurelhuda NM, Ahmed MF, Trovik TA, Astrom AN. Evaluation of oral health-related a quality of life among Sudanese schoolchildren using Child-OIDP inventory. Health Qual Life Outcomes. 2010;8:152. doi:10.1186/1477-7525-8-152

21. Yusuf H, Gherunpong S, Sheiham A, Tsakos G. Validation of an English version of the Child-OIDP index, an oral health-related quality of life measure for children. Health Qual Life Outcomes. 2006; 4:38.

22. Cortés-Martinicorena FJ, Rosel-Gallardo E, Artázcoz-Osés J, Bravo M, Tsakos G. Adaptation and val- idation for Spain of the child-oral impact on daily performance (C-OIDP) for use with adolescents. Med Oral Patol Oral Cir Bucal. 2010; 15(1):106-11.

23. Kida IA, Astrom AN, Strand GV, Masalu JR, Tsakos G. Psychometric properties and the prevalence, intensity and causes of oral impacts on daily permormance (OIDP) in a population of older Tanzanians. Health Qual Life Outcomes. 2006;4:56. doi:10.1186/14777525-4-56.

24. Ministerio de Salud. Prevalencia nacional de caries dental, fluorosis del esmalte y urgencia de tratamiento en escolares de 6 a 8, 10, 12 y 15 años, Perú, 20012002. Serie Informes Técnicos de Investigación Epidemiológica: No 05/050.Lima, Perú: Oficina General de Epidemiología, MINSA; 2005.

25. Tubert-Jeannin S, Pegon-Machat E, Gremeau-Richard C, Lecuyer M-M, Tsakos G. Validation of a French version of the Child-OIDP index. Eur J Oral Sc. 2005;113(5): 355-62.

26. Castro Rde A, Portela MC, Leão AT, de Vasconcelos MT. Oral health-related quality of life of 11-and 12-year-old public school children in Rio de Janeiro. Community Dent Oral Epidemiol. 2011; 39(4):336-44.

Recibido : 08/11/2014

Aceptado: 13/04/2015 